\title{
LTE/5G Mobil Terminaller için Log-Periyodik Tekniği Kullanılan Üç Bant Dört Elemanlı MIMO Anten Dizisi
}

\author{
Mehmet Yerlikaya $^{1 *}$, Seyfettin Sinan Gültekin ${ }^{2}$, Dilek Uzer $^{3}$ \\ ${ }^{1}$ Karamanoğlu Mehmetbey Üniversitesi, Mühendislik Fakültesi, Elektrik Elektronik Mühendisliği Bölümü, Karaman, Türkiye (ORCID: 0000-0000-0000-0000) \\ ${ }^{2}$ Konya Teknik Üniversitesi, Mühendislik Fakültesi, Elektrik Elektronik Mühendisliği Bölümü, Konya, Türkiye (ORCID: 0000-0000-0000-0000) \\ ${ }^{3}$ Konya Teknik Üniversitesi, Mühendislik Fakültesi, Elektrik Elektronik Mühendisliği Bölümü, Konya, Türkiye (ORCID: 0000-0000-0000-0000)
}

( $1^{\text {st }}$ International Conference on Computer, Electrical and Electronic Sciences ICCEES 2020 - 8-10 Ekim 2020)

(DOI: 10.31590/ejosat.803514)

ATIF/REFERENCE: Yerlikaya, M., Gültekin, S. S. \& Uzer, D. (2020). LTE/5G Mobil Terminaller için Log-Periyodik Tekniği Kullanılan Üç Bant Dört Elemanlı MIMO Anten Dizisi. Avrupa Bilim ve Teknoloji Dergisi, (Özel Sayı), 231-236.

$\ddot{O ̈ z}$

Bu çalışmada, günümüz teknolojisi olan LTE ya da $4.5 \mathrm{G}$ ile gelecek nesil olarak da bilinen beşinci nesil ( $5 \mathrm{G}$ ) mobil iletişimde kullanılan mobil terminaller için dört elemanlı çoklu giriş çoklu çıkış (MIMO) mimarisinde yeni bir yama anten dizisi sunulmuştur. Önerilen anten dizisinde yer alan her bir eleman log-periyodik yama şeklinde tasarlanmış ve karşılıklı gelen MIMO elamanları birbirinin özdeşi olacak şekilde yerleştirilmiştir. Bilindiği gibi, günümüzde yer alan mobil cihazlar oldukça kompakt yapıya sahiptirler. Bu cihazlarda yer alan anten sistemlerinin de bu boyutlara uygun olmaları gerektiğinden, önerilen antenin toplam boyutu $80 \times 150 \mathrm{~mm}^{2}$ ile standart bir akıllı cihazla eşdeğerdir. Önerilen antenin tasarımından sonra, 4.3 nispi geçirgenliğe ve $1.6 \mathrm{~mm}$ kalınlığa sahip FR4 substrat üzerine baskı devre (PCB) teknolojisi kullanılarak prototip üretimi de gerçekleştirilmiştir. Önerilen antenin ölçüm ve simülasyon değerleri arasında büyük oranda bir benzerlik elde edilmiştir. Önerilen dört elemanlı MIMO anten, 2.5-3 GHz, 3.3-4.2 GHz ve 4.95-6 GHz frekans bantları arasında $-10 \mathrm{~dB}$ ve altında ölçülen yansıma katsayısı değerleri ile üç bant ışıma yapmaktadır. Bu frekans bantları sırasıyla LTE 46, LTE 38 ve $5 \mathrm{G} \mathrm{C}$-bantlarını tam olarak kapsamaktadır. Belirtilen frekans bantlarında $16 \mathrm{~dB}$ ve üzerinde bir izolasyon seviyesi ile oldukça iyi bir karşı1ıklı kuplaj değerine sahiptir. Bununla birlikte, önerilen üç bantlı MIMO anten $2.6 \mathrm{GHz}, 3.6 \mathrm{GHz}$ ve $5.5 \mathrm{GHz}$ rezonans frekansı noktalarında sırasıyla $0.7 \mathrm{dBi}, 3.5 \mathrm{dBi}$ ve $4 \mathrm{dBi}$ olacak şekilde kazanç değerleri vardır. Ayrıca, önerilen MIMO anten dizisi belirtilen üç frekans bandı için de \%60 ve üzerinde bir verimliğe sahiptir. Son olarak, ışıma patternleri incelendiğinde, önerilen MIMO anten dizisinin her üç frekans noktasında da çok yönlü bir ışıma sergilediği görülmüştür.

Anahtar Kelimeler: Yama anten, 5G, LTE, MIMO, Log-periyodik, 6 GHz-alt1.

\section{Triple-Band Four-Element MIMO Antenna Array Using Log-Periodic Technique for LTE/5G Mobile Terminals}

\begin{abstract}
In this study, a new antenna array in four element multiple input multiple output (MIMO) architecture is presented for mobile terminals that used in today's mobile technology of LTE or $4.5 \mathrm{G}$ and fifth generation $(5 \mathrm{G})$ mobile communication, also known as the next generation. Each MIMO element in the proposed antenna array is designed as a log-periodic patch, and the corresponding MIMO elements are placed identical to each other. As is known, today's mobile devices have a very compact structure. Since the antenna systems in these devices must also comply with these dimensions, the overall size of the proposed antenna is $80 \times 150 \mathrm{~mm}^{2}$ which is equivalent to a standard smartphone. After designing the proposed antenna, prototype production was also carried out using printed circuit board (PCB) technology on FR4 substrate with 4.3 relative permeability and $1.6 \mathrm{~mm}$ thickness. There is a great agreement between the measured and simulated results of the proposed antenna. The proposed four-element MIMO antenna emits three bands with
\end{abstract}

\footnotetext{
* Sorumlu Yazar: Karamanoğlu Mehmetbey Üniversitesi, Mühendislik Fakültesi, Elektrik Elektronik Mühendisliği Bölümü, Karaman, Türkiye, ORCID: 0000-0000-0000-0000, myerlikaya@,kmu.edu.tr
} 
reflection coefficient values of $-10 \mathrm{~dB}$ or below between the frequency bands $2.5-3 \mathrm{GHz}, 3.3-4.2 \mathrm{GHz}$ and $4.95-6 \mathrm{GHz}$. These frequency bands fully cover the LTE 46, LTE 38 and 5G C-bands, respectively. It has a fairly good mutual coupling value with an izolation level of $16 \mathrm{~dB}$ or above in the specified frequency bands. However, the recommended tri-band MIMO antenna has gain values of $0.7 \mathrm{dBi}$, $3.5 \mathrm{dBi}$ and $4 \mathrm{dBi}$ at the $2.6 \mathrm{GHz}, 3.6 \mathrm{GHz}$ and $5.5 \mathrm{GHz}$ resonant frequency points, respectively. In addition, the proposed MIMO antenna array has an efficiency of $70 \%$ and above for all three specified frequency bands. Finally, when the radiation patterns were examined, it was seen that the proposed MIMO antenna array displayed a versatile radiation at all three frequency points.

Keywords: Patch antenna, 5G, LTE, MIMO, Log-periodic, Sub-6 GHz.

\section{Giriş}

1980’lerde analog iletişimin kullanıldığı 1. Nesil (1G) teknolojisi ile başlayan ve günümüzde dijital iletişimin kullanan 4. Nesil (4G) haberleşmeye kadar mobil iletişim teknolojisi, son derece hızlı bir gelişme ile her dönemde son kullanıcılara yeni hizmetler ve özellikler sunmuştur. Yüksek veri hızları, düşük bağlantı gecikmesi, düşük maliyet, düşük enerji kullanımı ve daha fazla kullanıcıya yönelik destek için artan talep, yeni nesil olarak adlandırılan beşinci nesil (5G) teknolojinin ortaya çıkmasının yolunu açtı [1]. 5G teknolojisinin ticarileştirilmesi için en önemli beklenti, frekans spektrum paylaşımı başta olmak üzere gerekli tüm standartların belirlenmesidir. Günümüzde, hücresel haberleşme olarak da bilinen mobil iletişim teknolojisi, daha çok $3 \mathrm{GHz}$ spektrumunun altındaki frekansları yaygın olarak kullanmaktadır. Bu durum özellikle $3 \mathrm{GHz}$ altındaki frekanslarda sıkışıklığa neden olmaktadır. 5G iletişim frekans spektrumu, fiziksel özellikler açısından $6 \mathrm{GHz}$ altı ve $6 \mathrm{GHz}$ üstü olarak iki aralıkta tanımlanmaktadır [2]. Tüm servis sağlayıcıların 2020 sonrası için birincil olarak kullanacakları bant 3.3-4.2 GHz frekans aralığında yer alan 5G C-bandıdır. Bu frekans bandının ilk aşamada tercih edilme sebebi ise, kanal kapasitesinin yüksek ve kapsama alanının geniş olmasıdır [3,4]. Buna ek olarak, 2500-2690 MHz frekans aralı̆̆ında tanımlı LTE Bant-38 ile 5150-5925 MHz aralığında yer alan LTE Bant-46, MIMO uygulamaları için 5G mobil terminallerde kullanılmak üzere önemli bir rekabet potansiyeline sahiptir [5].

Çoklu Giriş Çoklu Çıkış (MIMO), iki veya daha fazla antenin aynı anda hem iletim hem de radyo kanalı üzerinden alım için kullanılması anlamına gelir. MIMO teknolojisinde daha yüksek veri hızları elde etmek için çoklu yol kullanılır. Bunun neticesinde fazladan frekans bant genişliği kullanmadan bant aralığı artar, böylece farklı hizmetler için yüksek veri hızlarına duyulan ihtiyaca yönelik spektral verimlik artar. Bu nedenle MIMO teknolojisi, 5G hücresel iletişimin gerekli veri aktarım hızlarına ulaşmak için en umut verici teknolojidir [6,7].

Teorik olarak bir MIMO sisteminin kapasitesi, MIMO anten elemanlarının sayısı ile doğru orantılı artar, ancak aynı anten elemanlarının sınırlı alana sahip mobil cihazlarda birbirine yakın yerleştirilmesi neticesinde yüksek karşılıklı kuplaja neden olur. Bu durum da anten sisteminin performansını ve kapasitesini düşürür. Günümüz için -10 dB ve altında yer alan izolasyon seviyesi, $6 \mathrm{GHz}$ altı 5G MIMO anten sistemleri için kabul edilebilir seviye olarak kabul edilmektedir [8].

Literatürde 5G mobil terminalleri için bazı çoklu bant MIMO çalışmaları yer almaktadır [9-12]. Bu çalışmalardan 3.5/5.8GHz çift bantlı çalışma performansı elde eden [9]'da asimetrik olarak yansıtılmış yapı da kullanılmışıtır. Kuplaj beslemesi ve T-şekilli yuva elemanlarının kullanıldığı [10]'da ise, $6 \mathrm{GHz}$ altı çalışma için on elemanlı bir dizi şeklinde tasarlanan sistem çalışma bantları olan 3400$3800 \mathrm{MHz}$ ile 5150-5925 MHz frekans aralıklarında sırasıyla $-10 \mathrm{~dB}$ ve $-12.5 \mathrm{~dB}$ izolasyona sahiptir. Bir diğer çalışmada, her bir elemanın iki halka yapısından oluştuğu 8 elemanlı bir MIMO dizisi yer almaktadır [11]. 5G $6 \mathrm{GHz}$ altı mobil terminaller için önerilen bu MIMO dizisi, -6 dB'den düşük yansıma katsayısı kriteri altında $440 \mathrm{MHz}(3.38-3.82 \mathrm{GHz})$ ve $800 \mathrm{MHz}(4.80-5.60 \mathrm{GHz})$ bant genişliğine sahip iki bantta $15 \mathrm{~dB}$ üzerinde bir izolasyonla ışıma yapmaktadır. Son olarak [12]'de, 5G mobil terminalleri için iki uzun yan kenarı boyunca yerleştirilmiş iki simetrik dört öğeli alt diziden oluşan kompakt bir sekiz öğeli bir MIMO anten dizisi sunulmuştur. Önerilen MIMO anten dizisi, üç rezonans frekansı olan iki geniş bant kapsama ile 3 bantlı bir ışımaya ve elemanlar arasında $10 \mathrm{~dB}$ ve üzeri bir izolasyona sahiptir.

Bu çalışmada, mobil terminaller için karşılıklı ikişer simetrik elemandan oluşan toplam 4 elemanlı bir 5G MIMO anten dizisi önerilmiştir. Dizi elemanlarının her biri log-periyodik anten benzeri bir yamadan oluşan MIMO anten dizisi, $-10 \mathrm{~dB}$ yansıma katsayısı kriterinde üç bant çalışmaktadır. Antenin ışıma yaptı̆̆ı bantlar sırasıyla; LTE 38 bandını kapsayan 2500-3000 MHz, $5 \mathrm{G}$ n77 ve n78 bantlarını kapsayan 3.2-3.8 GHz ve LTE 46 numaralı bandı kapsayan 4.95-6 GHz frekans aralıklarında çalışmaktadır. MIMO elemanları arasında oldukça iyi bir karşılıklı kuplaj seviyesine sahip anten dizisinde tüm çalışma bantlarında $16 \mathrm{~dB}$ ve üzeri bir izolasyon değeri ölçülmüştür.

\section{Materyal ve Metot}

$\mathrm{Bu}$ çalışmada önerilen dört elemanlı MIMO anten dizisinin tasarım ve performans incelemeleri IE3D elektromanyetik simülasyon yazılımı ile elde edilmiştir [13]. Tasarım süreci sonrasında belirlenen antenin prototip üretimi baskı devre (PCB) teknolojisi ile gerçekleştirildikten sonra performans ölçümleri yansımasız oda içerisinde Keysight vektör network analizörü (VNA) ve EBTRO EAMS anten ölçüm sistemi ile yapılmıştır.

\subsection{MIMO Anten Geometrisi}

Mobil terminal uygulamaları için önerilen üç bantlı dört elemanlı 5G MIMO dizinin konfigürasyonu Şekil 1'de gösterilmektedir. 


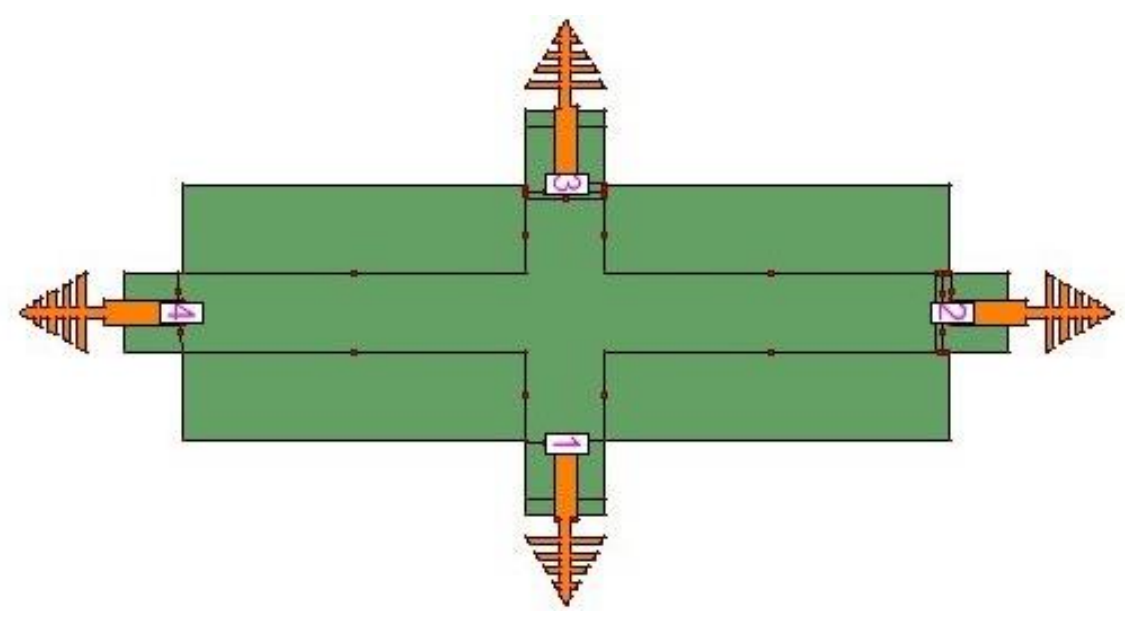

Şekil 1. Önerilen dört elemanlı MIMO anten dizisi konfigürasyonu

Şekilde görüldüğü üzere tüm elemanların yama düzlemleri birbiri ile eştir. Elemanlar arasındaki farklılık sadece dikdörtgen toprak düzlemlerindedir. Kısa kenar üzerinde yer alan elemanların toprak düzlemleri uzun kenar üzerindeki dizi elemanlarının toprak düzlemlerinden daha kısadır. Bu uzunluk farkı antenlerin besleme noktaları açısından da farklılık göstermesine neden olmuştur. MIMO anten sistemi için belirlenen toplam boyut $150 \times 80 \mathrm{~mm}^{2}$ ile standart bir akıllı telefon boyutudur.

\subsubsection{MIMO Elemanı Yaptst}

Yama antenlerde bant genişliğini artırmak için bazı yöntemler vardır. Literatürde kullanılan yöntemlerden birisi de log-periyodik yama kullanmaktır [14]. Önerilen dört elemanlı MIMO dizisinde yer alan her bir log-periyodik yama elemanının detaylı 2B geometrisi Şekil 2'de verilirken boyutları açıklamaları ile birlikte Tablo 1'de sunulmuştur.

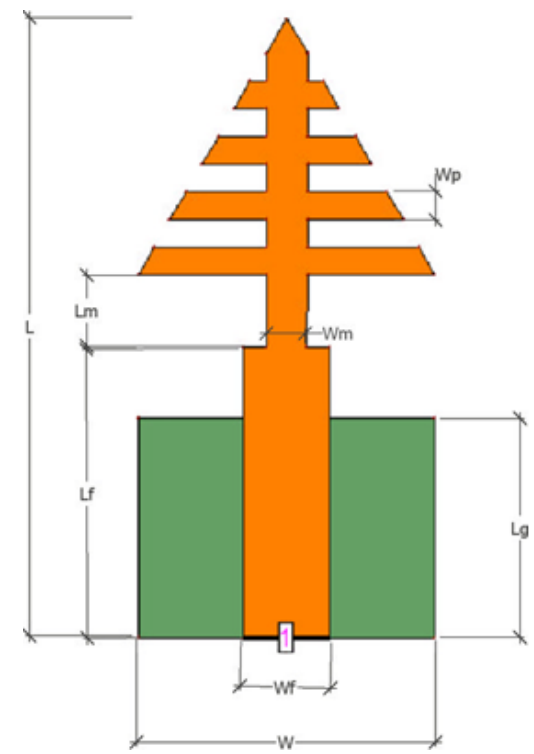

Şekil 2. Log-periyodik MIMO elemanı $2 B$ geometrisi

Şekilde görüldüğü üzere, önerilen anten yaması boyutu $9.3 \times 10.7 \mathrm{~mm}^{2}$ olan bir log-periyodik diziden oluşur. Bu log-periyodik yama, $3.1 \mathrm{~mm}$ genişliğinde ve $10.5 \mathrm{~mm}$ uzunluğunda bir mikroşerit besleme hattı ile beslenir. Ek olarak, mikroşerit besleme hattı ile yama arasındaki empedans eşleşmesini artırmak için 1,5 genişlik ve $2.7 \mathrm{~mm}$ uzunlukta bir şerit hat eklenmiştir.

Tablo 1. Log-periyodik MIMO elemanı boyutları ve açıklamaları

\begin{tabular}{|c|c|c|}
\hline Boyut & Açıklama & Büyüklük (mm) \\
\hline$W$ & MIMO eleman genişlĭği & 10.7 \\
\hline$L$ & MIMO eleman uzunluğu & 22.5 \\
\hline$W_{f}$ & MIMO elemanı besleme hattı genişliği & 3.1 \\
\hline$L_{f}$ & MIMO elemanı besleme hattı uzunluğu & 10.5 \\
\hline$W_{m}$ & Uyumlandırma hattı genişliği & 1.5 \\
\hline$L_{m}$ & Uyumlandırma hattı uzunluğu & 2.7 \\
\hline$W_{p}$ & Log-periyodik eleman kalınlığı & 1 \\
\hline$L_{g}$ & Toprak düzlemi uzunluğu & $8-10$ \\
\hline
\end{tabular}




\subsection{Anten Üretimi}

Önerilen MIMO antenin tasarım sürecinden sonra prototipi de üretilmiştir (Şekil 3). Anten tasarımında 4.3 dielektrik sabiti, 0.02 tanjant kaybı olan 1.6mm kalınlıkta bir FR4 substrat kullanılmıştır. Antenin üretimi LPKF Protomat türü cihazla PCB teknolojisi ile üretilmiştir. Baskı devre ile üretilen antene RF sinyal verebilmek ve ölçüm alabilmek için 50 ohm SMA konnektörler lehimlenmiştir.

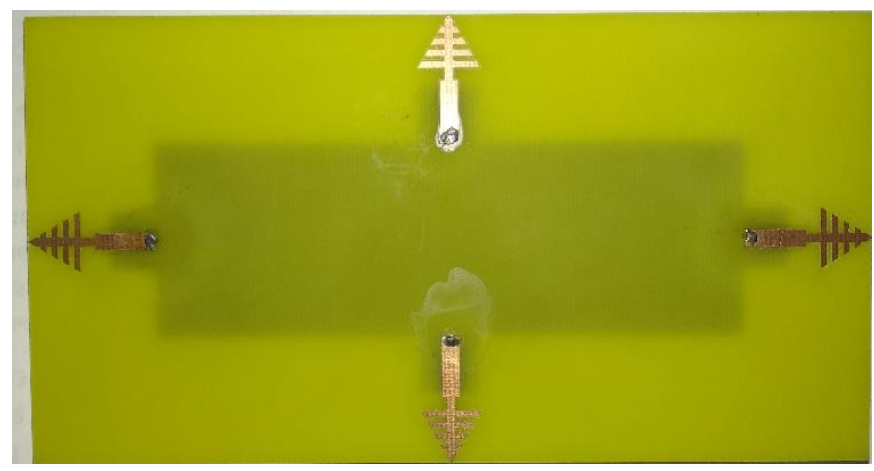

(a)

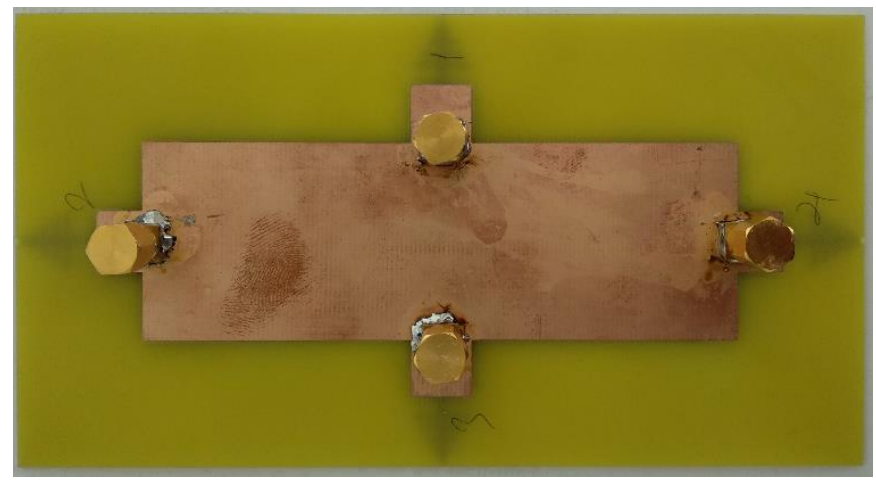

(b)

Şekil 3. Log-periyodik MIMO anten prototipi (a) üstten görünüm (b) alttan görünüm

\section{Araştırma Sonuçları ve Tartışma}

Önerilen log-periyodik elemanlı MIMO antenin yansıma katsayısı değerleri ölçüldüğünde, anten dizisinin -10 dB referansına göre 2.5-3 GHz, 3.3-4.2 GHz ve 4.95-6 GHz olacak şekilde üç bant çalıştığı görülmektedir. Bununla birlikte, ölçüm ve simülasyon Snn değerleri arasında büyük oranda benzerlik olduğu şekilden anlaşılmaktadır.

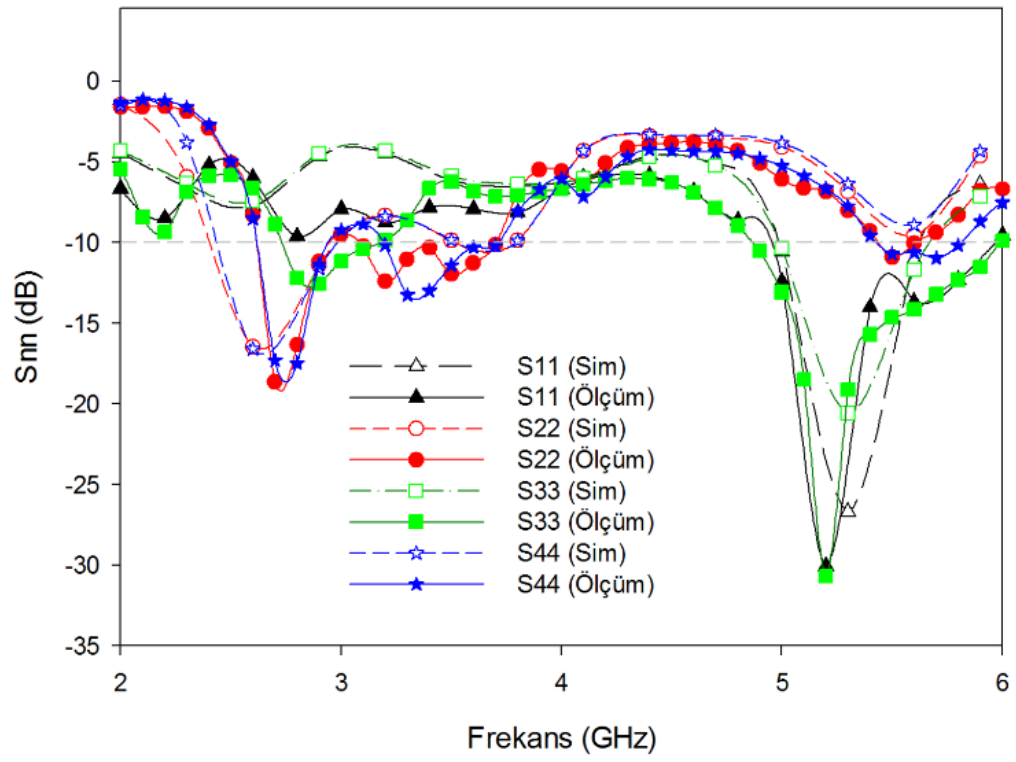

Şekil 4. Log-periyodik MIMO anten karşılaştırmalı ölçüm ve simülasyon yansıma katsayıları

Anten tasarımında tüm bantlarda $12 \mathrm{~dB}$ üzeri bir izolasyon seviyesi elde edilirken, ölçüm sonuçlarında izolasyon seviyesi tüm çalışma frekans aralıkları için $16 \mathrm{~dB}$ 'den daha iyi bir değer ölçülmüştür. 


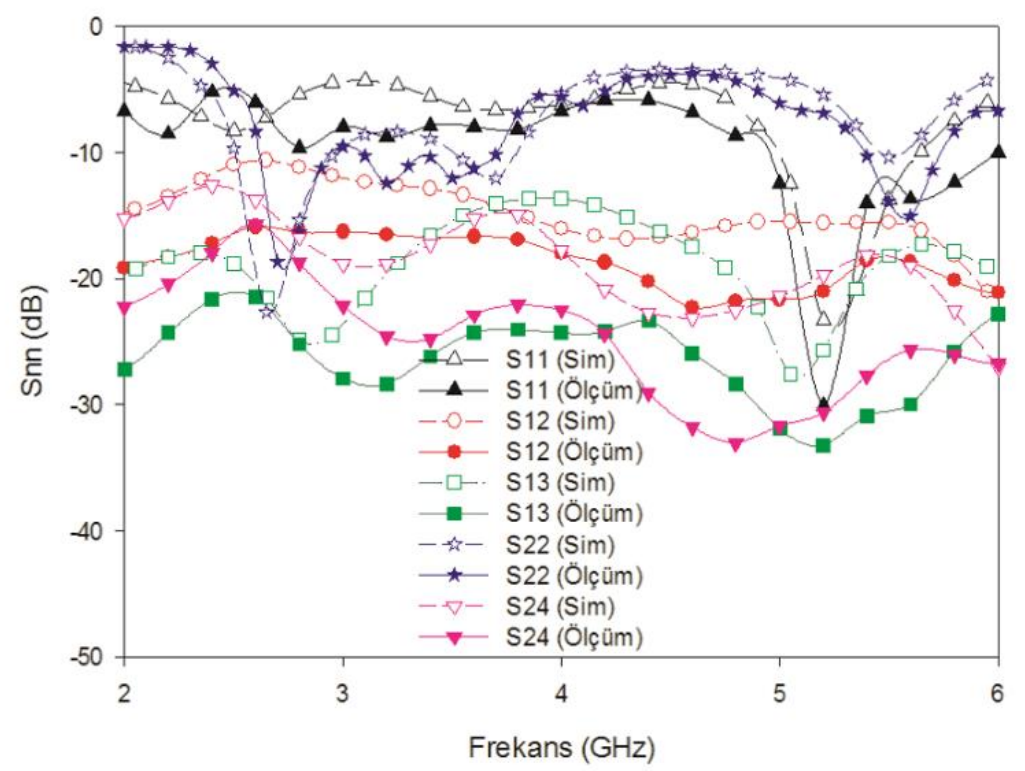

Şekil 5. Log-periyodik MIMO anten karşılaştırmalı ölçüm ve simülasyon iletim katsayıları

Önerilen MIMO antenin rezonans frekans noktaları olan $2.6 \mathrm{GHz}, 3.6 \mathrm{GHz}$ ve $5.5 \mathrm{GHz}$ için simüle edilen $\mathrm{xz}\left(\phi=0^{\circ}\right.$ veya Edüzlemi) ve yz $\left(\phi=90^{\circ}\right.$ veya H-düzlemi) düzlemleri için 2B 1şıma patternleri Şekil 6'da verilmektedir. Grafiklerde mor renkli çizgiler xz düzleminindeki değerleri, siyah renkli çizgiler ise yz düzlemindeki değerleri göstermektedir. Şekilden de anlaşılacağı gibi, MIMO anten dizisi her üç frekans noktası için de çok yönlü bir ışıma sergilemektedir. Belirtilen frekans noktaları için maksimum kazanç değerleri ise sırasıyla sırasıyla $0.07 \mathrm{dBi}, 1.9 \mathrm{dBi}$ ve $2.3 \mathrm{dBi}$ olarak ölçülmüştür.

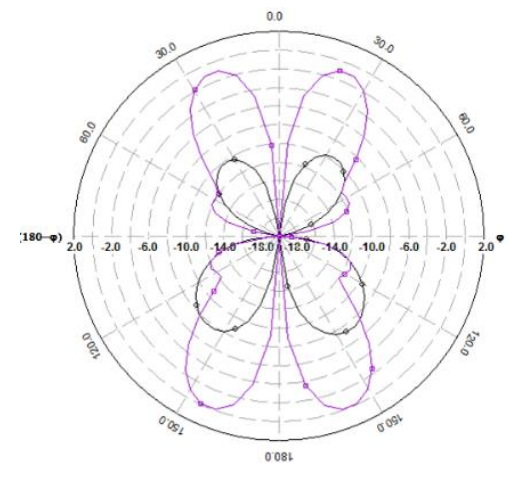

(a)

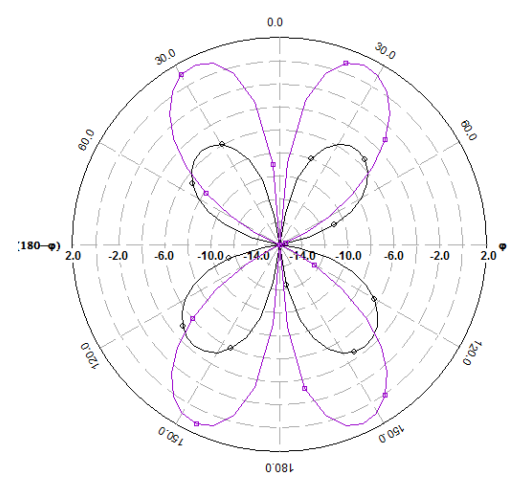

(b)

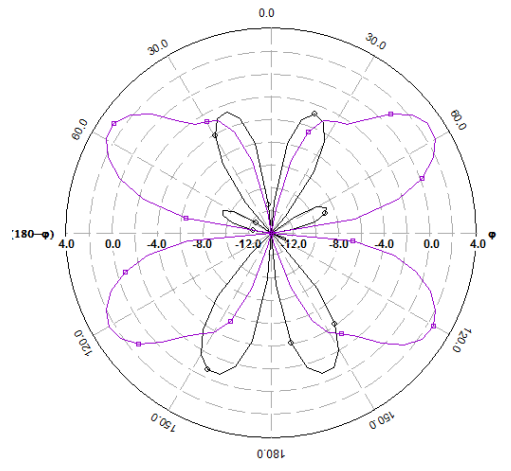

(c)

Şekil 6. Log-periyodik MIMO anten ışıma patternleri (a) $2.6 \mathrm{GHz}$ (b) $3.6 \mathrm{GHz}$ (c) $5.5 \mathrm{GHz}$

\section{Sonuç}

Sonuç olarak, bu çalışmada LTE ve $5 \mathrm{G}$ mobil cihazları için üç bant çalışabilen dört elemanlı bir MIMO anten dizisi önerilmiştir. Log-periyodik yama dizi elemanlarından oluşan MIMO antenin toplam boyutu, bir akıllı cihaz boyutu olacak şekilde $80 \times 150 \mathrm{~mm}^{2}$ olarak belirlenmiştir. Her bir MIMO dizi elemanının yama boyutu ise, $9.3 \times 10.7 \mathrm{~mm}^{2}$ olacak şekilde ayarlanmıştır. Kalınlığ $1.6 \mathrm{~mm}$ olan FR4 malzeme ile üretimi gerçekleştirilen antenin, çalışma bantları da 2.5-3 GHz, 3.3-4.2 GHz ve 4.95-6 GHz olarak ölçülmüştür. Tüm bu özellikleri ile önerilen MIMO anten, gelecek nesil $6 \mathrm{GHz}$ altı uygulamalar içim uygun bir çalışma olarak değerlendirilebilir.

\section{Kaynakça}

[1] Rappaport, T. S., Sun, S., Mayzus, R., Zhao, H., Azar, Y., Wang, K., Schulz, J. K., Samimi, M., and Gutierrez, F. (2013). Millimeter wave mobile communications for 5G cellular: It will work!. IEEE access, 1, 335-349.

[2] Lee, J., Tejedor, E., Ranta-aho, K., Wang, H., Lee, K. T., Semaan, E., Mohyeldin, E., Song, J., Bergljung, C., and Jung, S. (2018). Spectrum for 5G: Global status, challenges, and enabling technologies. IEEE Communications Magazine, 56(3), 12-18. J. Lee, E. Tejedor, K. Ranta-aho, H. Wang, K. T. Lee, E. Semaan, S. Jung, "Spectrum for 5G: global status, challenges, and enabling Technologies”, IEEE Communications Magazine, vol. 56 (3), pp. 12-18, 2018.

[3] Marcus, M. J. (2015). 5G and" IMT for 2020 and beyond"[Spectrum Policy and Regulatory Issues]. IEEE Wireless Communications, 22(4), 2-3. 
[4] Saxena, S., Kanaujia, B. K., Dwari, S., Kumar, S., and Tiwari, R. (2018). MIMO antenna with built-in circular shaped isolator for sub-6 GHz 5G applications. Electronics letters, 54(8), 478-480.

[5] Bajracharya, R., Shrestha, R., Zikria, Y. B., and Kim, S. W. (2018). LTE in the unlicensed spectrum: A survey. IETE Technical Review, 35(1), 78-90.

[6] Kildal, P. S., and Rosengren, K. (2004). Correlation and capacity of MIMO systems and mutual coupling, radiation efficiency, and diversity gain of their antennas: simulations and measurements in a reverberation chamber. IEEE Communications Magazine, 42(12), 104-112.

[7] Andrews, J. G., Buzzi, S., Choi, W., Hanly, S. V., Lozano, A., Soong, A. C., and Zhang, J. C. (2014). What will 5G be?. IEEE Journal on selected areas in communications, 32(6), 1065-1082.

[8] Li, Y., Luo, Y., and Yang, G. (2019). High-isolation 3.5 GHz eight-antenna MIMO array using balanced open-slot antenna element for $5 \mathrm{G}$ smartphones. IEEE Transactions on Antennas and Propagation, 67(6), 3820-3830.

[9] Wong, K. L., Lin, B. W., and Li, B. W. Y. (2017). Dual-band dual inverted-F/loop antennas as a compact decoupled building block for forming eight 3.5/5.8-GHz MIMO antennas in the future smartphone. Microwave and Optical Technology Letters, 59(11), 27152721.

[10]Li, Y., Luo, Y., and Yang, G. (2018). Multiband 10-antenna array for sub-6 GHz MIMO applications in 5-G smartphones. IEEE Access, 6, 28041-28053.

[11]Hu, W., Qian, L., Gao, S., Wen, L. H., Luo, Q., Xu, H., Liu, X., Liu, Y., and Wang, W. (2019). Dual-band eight-element MIMO array using multi-slot decoupling technique for 5G terminals. IEEE Access, 7, 153910-153920.

[12]Wang, H., Zhang, R., Luo, Y., \& Yang, G. (2020). Compact Eight-Element Antenna Array for Triple-Band MIMO Operation in 5G Mobile Terminals. IEEE Access, 8, 19433-19449.

[13] IE3D, HyperLynx 3D EM, Mentor Graphics.

[14] Yerlikaya, M., Gültekin, S. S., \& Dilek, U. Z. E. R. (2020). A novel design of a compact wideband patch antenna for sub-6 GHz fifth-generation mobile systems. International Advanced Researches and Engineering Journal, (View), 129-133. 